\title{
EDITORIAL
}

\section{A day off in Denmark}

\author{
A day off in the middle of the schedule of a recent microbial ecology conference highlighted \\ the importance of taking time out.
}

As you may well have seen from our Twitter feed (@NatureRevMicro), we recently attended the International Society for Microbial Ecology (ISME) conference in Copenhagen, Denmark. The number of participants at a particular conference depends on many different variables, but overall, it seems reasonable to conclude that the attendance at a flagship meeting in a specific research area tends to reflect the health of that field, and this was the case with the ISME meeting. As a discipline, microbial ecology has been undergoing an impressive renaissance over the past decade, and it continues to go from strength to strength. From its humble beginnings (there were fewer than 500 attendees at the first ISME conference more than 30 years ago), this biannual meeting has grown along with the field. From the late 1990s onwards - perhaps reflecting the time when metagenomic analyses of environmental samples began to be used more widely - attendance increased substantially, and this time around the conference attracted more than 2,200 registered participants, making it the most successful ISME conference to date.

There are of course other meetings that can attract a similar number of microbiologists, and indeed some that attract greater numbers. What makes the ISME meeting unusual, however, is that the conference schedule includes a full day's break in the middle. Many other microbiology conferences include the chance to join an afternoon or evening excursion or social event, or schedule in some free time between the scientific sessions to foster discussion and interaction, but to our knowledge a full 'rest day' is unique. It also goes against the prevailing trend for many scientific conferences to pare down the schedules to cut costs. For example, the American Society for Microbiology's annual General Meeting recently underwent a reorganization that has resulted in the schedule being shortened by half a day, partly to reduce accommodation costs. A full day off is therefore a rare event, but it is regarded as sacred for this biannual jamboree, even given the financial implications of having to book the venue for 5 days when it is only in use for 4 of those.

Although it may be unusual, all of the participants that we spoke to at the meeting were enthusiastic supporters of this break in proceedings. Attendance at a large conference these days can be a pretty frenetic experience, with the pressure of switching between many parallel sessions and squeezing through the throngs at the posters, and for many people a day to reflect on, and process, the information from the first two days was very welcome. For others, it signalled the rare opportunity to actually see more than the inside of the conference centre and hotel room. To attract attendees, microbiology conferences can be held in some of the most fascinating cities in the world, but paradoxically, many attendees - particularly senior investigators, who can be on the road for a large proportion of every year - get no chance to explore. The organized tours of Copenhagen and the surrounding areas were therefore a popular draw. The full day's break also contributed to the family-friendly nature of this conference, with many taking the chance to bring their partners and/or children. One of the main aims of the break, in addition to the chance to explore the local area, is of course to afford the opportunity to have a full- or half-day meeting with colleagues to discuss ongoing collaborations or, perhaps more importantly, to set up new ones. Hopefully, many participants took the chance to do so. For many, though, old habits die hard, and the day was simply spent catching up on email and liaising with the lab back at home.

Given this enforced break in the meeting, it is interesting to note that while there, we encountered a smattering of microbial ecologists who are currently enjoying an enforced break from the pressures of running a lab by taking a period of sabbatical leave, and all of whom were wondering why they hadn't done so before. In today's publish-or-perish climate, a life in science can be relentless, and researchers often lack the time to take a step back from their specific area and look at the bigger picture. The benefits of the break in the meeting were obvious when the reinvigorated crowds poured in the next day, and the benefits of taking time out from your research, even if for just a day or two, can be equally energizing. So heed our advice - and take a day off!

\section{FURTHER INFORMATION}

ISME: http://www.isme-microbes.org

Nature Reviews Microbiology on Twitter: https://twitter.com/NatureRevMicro ALL LINKS ARE ACTIVE IN THE ONLINE PDF 